\title{
Commentary Host immune response in sepsis due to ventilator-associated pneumonia: how is it different?

\author{
Eirini Christaki
}

'Division of Infectious Diseases, The Warren Alpert Medical School of Brown University, The Miriam Hospital, 164 Summit Avenue, Providence, RI 02906, USA

Corresponding author: Eirini Christaki, eirini.christaki@gmail.com

Published: 7 December 2009

Critical Care 2009, 13:1009 (doi:10.1186/cc8174)

This article is online at http://ccforum.com/content/13/6/1009

(C) 2009 BioMed Central Ltd

See related research by Pelekanou et al., http://ccforum.com/content/13/6/R172

\begin{abstract}
Current evidence regarding potentially different host response mechanisms in sepsis according to the type of initiating infection is sporadic. It is possible that alterations in cell populations, variations in effector molecules, and the degree of apoptosis differ between sepsis caused by ventilator-associated pneumonia (VAP) and nonVAP sepsis. VAP is one of the most common infections and leading causes of sepsis in the intensive care unit, and mortality remains high. A better understanding of the unique pathophysiologic features of VAP is needed in order to develop interventions that target those specific pathways.
\end{abstract}

Ventilator-associated pneumonia (VAP) develops commonly in mechanically ventilated patients and is a major cause of morbidity and mortality in the intensive care unit. In a study published in this issue of Critical Care, Pelekanou and colleagues [1] investigated the differences in innate and adaptive immune responses in 36 septic patients with VAP and 32 patients with sepsis due to other infections, like pyelonephritis, bacteremia, intra-abdominal infection, and community- and hospital-acquired pneumonia. There was evidence of a more pronounced immunoparalysis in patients with VAP than in those with other bacterial infections. This was supported by the decreased number of $\mathrm{CD}^{+} / \mathrm{CD} 4^{+}$ cells, the increase in monocyte apoptosis, and the lower release of pro-inflammatory cytokines, namely tumor necrosis factor-alpha and interleukin-6, from monocytes after stimulation with lipopolysaccharide (LPS) in the group of patients with VAP.

It is known that anergic monocytes from patients with septic shock showed increased susceptibility to apoptosis when compared with monocytes from normal hosts [2]. Patients with VAP are more compromised due to various factors like critical illness, malnutrition, invasive interventions, and the loss of anatomic defense mechanisms, some of which may contribute to monocyte unresponsiveness or lymphocyte depletion. The authors report that endotracheal intubation in septic patients without VAP was not independently associated with similar numeric and functional alterations in lymphocytes and monocytes. Nevertheless, the study might have been underpowered to detect such differences.

Additionally, one important finding of the study [1] was the observation that septic patients with VAP whose monocytes failed to adequately respond to monocyte stimulation had decreased survival rates when compared with those with an increased cytokine release from monocytes. A similar trend was observed in non-VAP-related sepsis, but it was not statistically significant. Previously published work from this group had demonstrated that early monocyte apoptosis was linked to survival advantage in patients with sepsis due to VAP [3]. What remain to be determined are whether a separate mechanism associated with monocyte anergy and enhanced apoptosis exists in VAP-related sepsis and how is it related to mortality.

One concept that may be useful in trying to answer this question is the development of endotoxin tolerance. Monocytes exposed to low doses of LPS exhibit a decreased responsiveness to subsequent stimulation by endotoxin [4]. Endotoxin tolerance has been considered a paradigm of immunoparalysis [5], which is present not only in sepsis but also in systemic inflammatory response syndrome and other diseases like cystic fibrosis and acute coronary syndrome $[6,7]$. Endotoxin tolerance could support the theory of VAP pathogenesis that is embraced by the authors. Gradual exposure of the host to increasing bacterial inocula originating from aspiration of oropharyngeal flora [8] may contribute to a state of immunoparalysis through the mechanism of endotoxin tolerance. However, the clinical 
implications of endotoxin tolerance continue to be elusive [4]. Although endotoxin tolerance has been implicated in increased susceptibility to secondary infections, a number of studies in experimental models of sepsis have exhibited a protective role of endotoxin tolerance [9-11].

Using a model similar to that of endotoxin tolerance [12], the authors attempted to mimic VAP pathogenesis by using augmenting concentrations of Gram-negative bacteria to sequentially stimulate ex vivo peripheral blood mononuclear cells (PBMCs) isolated from healthy volunteers and assessed their apoptosis parameters [1]. An increase in CD14 monocyte apoptosis was observed when compared with nonstimulated PBMCs and PBMCs that had only a unique bacterial challenge with the highest concentration of bacterial inoculum used.

Moreover, a question raised by the results is whether lymphocyte depletion, monocyte apoptosis, and monocyte anergy in VAP are immunoparalysis markers that could be used as prognostic factors or are underlying dysregulations that contribute to the pathogenesis of VAP. Although patients in the two groups did not differ significantly in terms of age, disease severity, underlying conditions, diabetes mellitus, corticosteroid use, the presence of other recent infections, or additional factors that may affect the immune response to sepsis, the duration of critical illness prior to enrollment was not reported. It would be reasonable to expect a more frequent occurrence of longer hospitalization, surgery, trauma, neurosurgical conditions, or other critical illness prior to the development of septic shock in the group of patients with VAP. Thus, it is hard to know whether any of the above-mentioned factors independently contributed to the observed monocyte unresponsiveness, monocyte apoptosis, and CD3/ CD4 cell decrease in this group, and more work is needed before those changes are attributed solely to VAP.

\section{Competing interests}

The author declares that they have no competing interests.

\section{References}

1. Pelekanou A, Tsagkaris I, Kotsaki A, Karagianni V, Giamarellou H, Armaganidis A, Giamarellos-Bourboulis EJ: Decrease of CD4 lymphocytes and apoptosis of CD14 monocytes are characteristic alterations in sepsis caused by ventilator-associated pneumonia: results from an observational study. Crit Care 2009, 13:R172.

2. Williams MA, Withington S, Newland AC, Kelsey SM: Monocyte anergy in septic shock is associated with a predilection to apoptosis and is reversed by granulocyte-macrophage colonystimulating factor ex vivo. J Infect Dis 1998, 178:1421-1433.

3. Giamarellos-Bourboulis EJ, Routsi C, Plachouras D, Markaki V, Raftogiannis M, Zervakis D, Koussoulas V, Orfanos S, Kotanidou A, Armaganidis A, Roussos C, Giamarellou H: Early apoptosis of blood monocytes in the septic host: is it a mechanism of protection in the event of septic shock? Crit Care 2006, 10:R76.

4. Cavaillon JM, Adib-Conquy M: Bench-to-bedside review: endotoxin tolerance as a model of leukocyte reprogramming in sepsis. Crit Care 2006, 10:233.

5. Biswas SK, López-Collazo E: Endotoxin tolerance: new mechanisms, molecules and clinical significance. Trends Immunol 2009, 30:475-487.
6. del Fresno C, Soler-Rangel L, Soares-Schanoski A, Gómez-Piña V, González-León MC, Gómez-García L, Mendoza-Barberá E, Rodríguez-Rojas A, García F, Fuentes-Prior P, Arnalich F, LópezCollazo E: Inflammatory responses associated with acute coronary syndrome up-regulate IRAK-M and induce endotoxin tolerance in circulating monocytes. J Endotoxin Res 2007, 13: 39-52.

7. del Fresno C, Gómez-Piña V, Lores V, Soares-Schanoski A, Fernández-Ruiz I, Rojo B, Alvarez-Sala R, Caballero-Garrido E, García F, Veliz T, Arnalich F, Fuentes-Prior P, García-Río F, López-Collazo $E:$ Monocytes from cystic fibrosis patients are locked in an LPS tolerance state: down-regulation of TREM-1 as putative underlying mechanism. PLOS One 2008, 3:e2667.

8. Safdar N, Crnich CJ, Maki DG: The pathogenesis of ventilatorassociated pneumonia: its relevance to developing effective strategies for prevention. Respir Care 2005, 50:725-739; discussion 39-41.

9. Murphey ED, Fang G, Sherwood ER: Endotoxin pretreatment improves bacterial clearance and decreases mortality in mice challenged with Staphylococcus aureus. Shock 2008, 29:512518.

10. Murphey ED, Fang G, Varma TK, Sherwood ER: Improved bacterial clearance and decreased mortality can be induced by LPS tolerance and is not dependent upon IFN-gamma. Shock 2007, 27:289-295.

11. Wheeler DS, Lahni PM, Denenberg AG, Poynter SE, Wong HR, Cook JA, Zingarelli $B$ : Induction of endotoxin tolerance enhances bacterial clearance and survival in murine polymicrobial sepsis. Shock 2008, 30:267-273.

12. del Fresno C, García-Rio F, Gómez-Piña V, Soares-Schanoski A, Fernández-Ruíz I, Jurado T, Kaijii T, Shu C, Marín E, Gutierrez del Arroyo A, Prados C, Arnalich F, Fuentes-Prior P, Biswas SK, López-Collazo E: Potent phagocytic activity with impaired antigen presentation identifying lipopolysaccharide-tolerant human monocytes: demonstration in isolated monocytes from cystic fibrosis patients. J Immuno/ 2009, 182:6494-6507. 